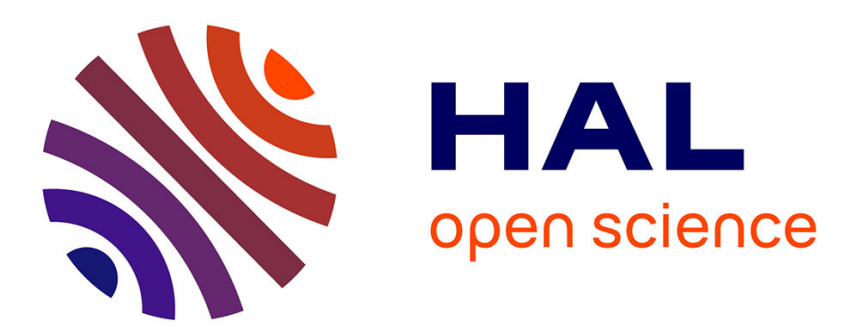

\title{
Evolution de la morphologie claviculaire au sein du genre Homo. Conséquences architecturales et fonctionnellessur la ceinture scapulaire
}

\author{
Jean-Luc Voisin
}

\section{- To cite this version:}

Jean-Luc Voisin. Evolution de la morphologie claviculaire au sein du genre Homo. Conséquences architecturales et fonctionnellessur la ceinture scapulaire. L'anthropologie, 2001, 105, pp.449-468. halshs-00351236

\author{
HAL Id: halshs-00351236 \\ https://shs.hal.science/halshs-00351236
}

Submitted on 8 Jan 2009

HAL is a multi-disciplinary open access archive for the deposit and dissemination of scientific research documents, whether they are published or not. The documents may come from teaching and research institutions in France or abroad, or from public or private research centers.
L'archive ouverte pluridisciplinaire HAL, est destinée au dépôt et à la diffusion de documents scientifiques de niveau recherche, publiés ou non, émanant des établissements d'enseignement et de recherche français ou étrangers, des laboratoires publics ou privés. 


\title{
Évolution de la morphologie claviculaire au sein du genre Homo. Conséquences architecturales et fonctionnelles sur la ceinture scapulaire
}

\author{
Jean-Luc Voisin*
}

\begin{abstract}
Résumé - La clavicule est un os qui a étẻ peu étudié en anthropologie et paléoanthropologie malgré son importance fonctionnelle. Le travail présenté ici est une étude des courbures claviculaires chez les hominoïdea actuels et chez quelques hominidés fossiles (Homo habilis, Homo ergaster, Homo antecessor et néandertaliens). La morphologie claviculaire en vue supérieure traduit essentiellement les capacités d'élévation du bras de l'individu, alors que la morphologie claviculaire en vue postérieure informe sur la position de la scapula par rapport au thorax. Les clavicules fossiles, notamment les néandertaliennes, montrent, en vue supérieure, une morphologie proche de celle de l'homme moderne. Les capacités de mouvement du bras chez ces hominidés devaient donc être similaires à celles de l'homme actuel. En vue postérieure, les clavicules de ces fossiles présentent une double courbure. Ces caractéristiques traduisent chez ces hominidés une scapula plus haute par rapport au thorax que chez l'homme moderne. (c) 2001 Éditions scientifiques et médicales Elsevier SAS
\end{abstract}

\section{clavicule / hominoïdea / Néandertal / Homo erectus / épaule}

\begin{abstract}
Evolution of the Clavicular Morphology within the Genus Homo. Architectural and Functional Consequences on the Shoulder Girdle. The clavicle is the less studied shoulder bone from the point of view of comparative anatomy in spite of its importance for the movements of the upper limbs. In this study we have compared the clavicle curvature between extant hominoids and some fossils (Homo habilis, Homo ergaster, Homo antecessor and Neandertal). The curvature in superior view shows the capacity of arm elevation. In posterior view, the curves show the position of the scapula in regard to the thorax. In superior view, there is no real difference between fossils and modern man. This means that human fossils clavicles, in peculiar neandertal one, do not presented a more $\mathrm{S}$ shaped morphology than those of modern human. In posterior view, the clavicle of fossil men shows that their scapula was situated higher on the thorax than that of modern human. (C) 2001 Éditions scientifiques et médicales Elsevier SAS
\end{abstract}

clavicle / hominoidea / Neandertal / Homo erectus / shoulder

\section{Introduction}

L'épaule est un complexe anatomique particulier car elle est constituée de plusieurs articulations qui interviennent en synergie lors des mouvements du bras, en faisant une part très importante aux structures dites "molles" (muscles et ligaments) les mieux adaptées à supporter des forces de tension. En effet, l'épaule n'est constituée que de trois os : la scapula (omoplate), la clavicule et la partie proximale de l'humérus, et de plus de vingt muscles (le nombre exact dépendant de l'espèce de primate). Ainsi, pour comprendre l'architecture et la biomécanique de l'épaule chez les formes humaines fossiles, il est nécessaire d'étudier précisément chacune des structures osseuses et ses corrélations avec les parties molles chez les primates actuels.

Bien que l'épaule ne soit constituée que de trois os, on constate une disparité importante quant au nombre d'étude ayant porté sur chacun d'entre eux. La grande majorité des travaux porte sur la scapula, contrairement à la clavicule qui est le parent pauvre de la paléoanthropologie, de l'anthropologie et surtout de l'anatomie comparée.

* Correspondance et tirés à part. jeanlucv@òmnhn.fr

Institut de paléontologie humaine, 1 rue R.-Panhard, 75013 Paris, France. 
Tableau 1. Effectifs de clavicules actuelles étudiées. Les pièces proviennent dans la mesure du possible d'individus sauvages.

Table I. Number of modern clavicle studied. Whenever possible they come from non captive specimen.

\begin{tabular}{lc}
\hline Espèces (abréviation) & Clavicule \\
\hline Homo sapiens sapiens $(\mathrm{Hm})$ & 33 \\
Pan troglodytes $(\mathrm{Pt})$ & 26 \\
Pan paniscus $(\mathrm{Pp})$ & 19 \\
Gorilla gorilla $(\mathrm{Gor})$ & 33 \\
Pongo pygmaeus $(\mathrm{Oo})$ & 24 \\
Hylobates $\mathrm{sp} .(\mathrm{Gb})$ & 22 \\
Papio hamadryas $(\mathrm{Ba})$ & 28 \\
\hline
\end{tabular}

Tableau II. Pièces fossiles étudiées ; *pièces originales.

Table II. Fossil clavicles studied; *original remains.

\begin{tabular}{lll}
\hline Espèces & \multicolumn{1}{c}{ Individus } & \multicolumn{1}{c}{ Clavicule } \\
\hline Neandertal & Régourdou & Droite et gauche \\
& Kebara & Droite et gauche \\
& La Ferrassie I* & Droite et gauche \\
& Krapina 4 & Gauche \\
& Neanderthal & Droite \\
Homo antecessor & Gran Dolina ATD6-50 & Droite \\
Homo ergaster & KNM-WT 15000 & Droite et gauche \\
Homo habilis & OH 48 & Gauche \\
\hline
\end{tabular}

Le travail présenté ici a pour but de comparer les clavicules des représentants fossiles du genre Homo avec celles de l'homme moderne. En fonction des résultats obtenus par comparaison avec différents primates, des interprétations architecturales et fonctionnelles seront proposées.

Les premières études sur la clavicule, bien que peu nombreuses, étaient essentiellement anthropologiques et avaient pour objectif de déterminer les différences et les similitudes entre les groupes humains (Parson, 1917 ; Kleiweg de Zwaan, 1931 ; Terry, 1932 ; Apostolakis, 1934 ; Olivier, 1951a, 1951b, 1954, 1955 ; Olivier et al., 1954 ; Olivier et Capliez, 1957 ; Ray, 1959 ; Jit et Kaur, 1986). Les travaux portant sur la fonction précise de cet os (Cave, 1961 ; Jenkins, 1974 ; Jenkins et al., 1978 ; Ljunggren, 1979 ; Harrington et al., 1993) ou ceux sur l'anatomie comparée, notamment au sein des primates (Schultz, 1930 ; Olivier, 1953 ; Voisin 2000a, $2000 \mathrm{~b}$ ) sont nettement moins nombreux. Cette lacune est d'autant plus étonnante que la clavicule présente un rôle fondamental dans les mouvements du bras. Elle permet, en effet, au membre supérieur de réaliser des mouvements de grande amplitude en dehors du plan parasagittal. En d'autres termes, elle permet la manipulation et l'arboricolisme. Ainsi, les transformations qui ont affecté la clavicule au cours de l'évolution humaine ont été accompagnées de modifications comportementales.

L'absence de données comparatives limite l'étude des pièces fossiles aux seules descriptions, exception faite de quelques travaux tels ceux de Heim (1974, 1982a, 1982b), Vandermeersch et Trinkaus (1995) et Sankhyan (1997).

\section{Matériel et méthodes}

\subsection{Matériel}

Le matériel étudié est composé de clavicules d'homme moderne, provenant de différentes

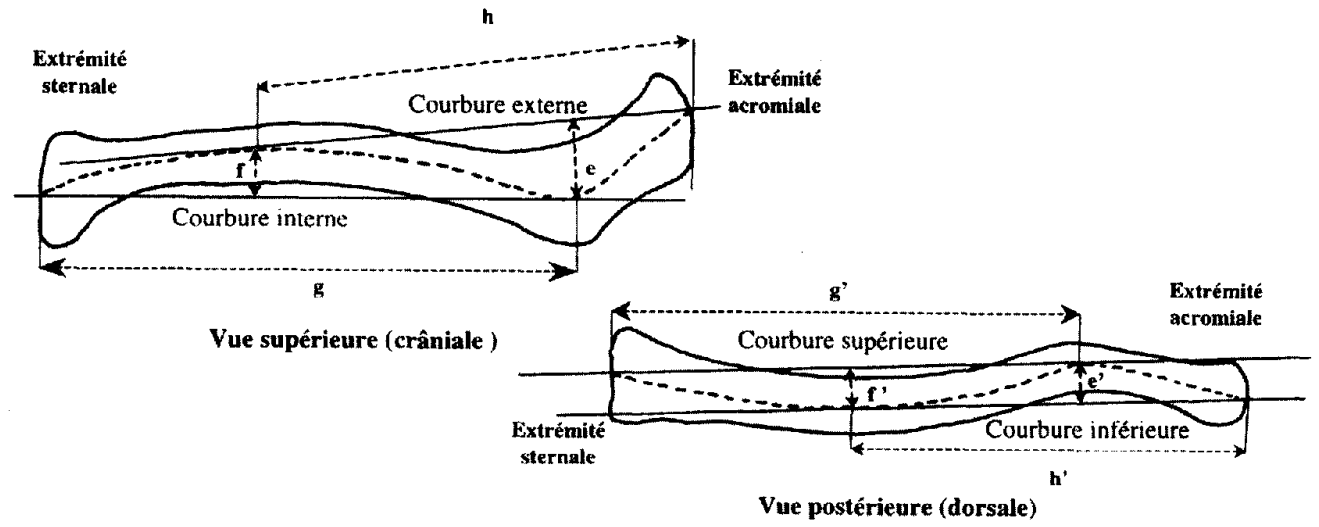

Figure 1. Détermination des arcs de courbures selon Olivier (1951a). Clavicule droite de Pan troglodytes.

Figure 1. Determination of the clavicle curves (Olivier, 1951a). Right clavicle of Pan troglodytes. 
Tableau III. Caractéristiques des courbures interne et externe des espèces actuelles étudiées ici.

Table III. Characteristics of the clavicule curves in superior view (internal an external).

\begin{tabular}{lcccccc}
\hline & \multicolumn{3}{c}{ Courbure interne } & \multicolumn{3}{c}{ Courbure externe } \\
\hline Espèce (nombre de pièces) & moyenne & écart-type & variance & moyenne & écart type & variance \\
Homo sapiens sapiens (33) & 12,6 & 2,5 & 6,3 & 16,1 & 2,7 & 7,3 \\
Pan troglodytes (26) & 8,1 & 2,8 & 7,8 & 15,4 & 3,0 & 9,0 \\
Pan paniscus (19) & 7,5 & 2,0 & 4,0 & 14,8 & 2,6 & 6,8 \\
Gorilla gorilla (33) & 3,3 & 1,9 & 3,6 & 12,6 & 3,1 & 9,6 \\
Pongo pygmaeus (24) & 5,8 & 2,1 & 4,4 & 9,1 & 2,3 & 5,3 \\
Hylobates sp. (22) & 12,6 & 2,8 & 7,8 & 5,3 & 2,3 & 5,3 \\
Papio hamadryas (28) & 2,2 & 1,2 & 1,4 & 14,1 & 2,8 & 7,8 \\
\hline
\end{tabular}

régions du monde, de gorille, de chimpanzé commun, de bonobo, d'orang-outan, de gibbon, de babouin (tableau $I$ ) et de fossiles (tableau II) contenant des pièces attribuées à Homo habilis, Homo ergaster, Homo antecessor et à des néandertaliens. Ce matériel provient des collections du Laboratoire d'Anthropologie Biologique du Musée de l'Homme, des Laboratoires d'Anatomie Comparée et des Mammifères et Oiseaux du Muséum National d'Histoire Naturelle, du Musée Royal d'Afrique Centrale de Tervuren (Belgique) et du Mammals Group du Natural History Museum de Londres (Royaume-Uni).

Les espèces de primates ont été choisies en fonction de leur mode locomoteur dominant et de leur place dans la phylogénie.

Le terme grand singe regroupe les chimpanzés communs, les bonobos, les gorilles et les orangs-outans, les trois premiers constituant les grands singes africains. Le terme hominoïde regroupe les hommes, les grands singes et les gibbons (Groves, 1993a). Sous l'appellation Hylobates sp., nous regroupons des clavicules appartenant aux sous-genres Nomascus et Hylobates s. str. En effet, ces deux sous-genres sont suffisamment proches pour que l'hybridation soit possible entre eux (Groves, 1993b).

\subsection{Méthodes}

Dans ce travail, seules les courbures claviculaires seront étudiées. Ces dernières, projetées dans deux plans perpendiculaires à l'aide d'un dioptographe, se décomposent en quatre courbures élémentaires, soit deux par plan (figure 1). Ces deux plans peuvent être assimilés, l'un à la vue supérieure, l'autre à la vue postérieure.

L'arc de courbure moyen est estimé, selon la méthode d'Olivier (1951a), en calculant le rap- port entre la longueur de la corde et sa hauteur maximale (figure 1).

\section{Vue supérieure}

- La courbure acromiale correspond à la courbure externe : e / h. 100 .

- La courbure sternale correspond à la courbure interne : f/g. 100.

\section{Vue postérieure}

- La courbure acromiale correspond à la courbure inférieure : e'/ h'.100.

- La courbure sternale correspond à la courbure supérieure : f'/g'.100.

La description et la répartition des données ont été analysées à l'aide du logiciel Systaw5. La représentation graphique de l'amplitude de variation pour chaque variable est donnée par un diagramme représentant la valeur moyenne et $+/$ - deux fois l'écart type.

\section{Résultats}

\subsection{Les courbures en vue supérieure}

\subsubsection{Primates actuels}

En vue supérieure, les courbures claviculaires permettent de différencier trois groupes (figure 2) :

- Les gorilles et les babouins qui présentent des clavicules caractérisées par un développement inégal des courbures. La courbure externe est très marquée contrairement à l'interne qui peut même être absente (figure 3 et tableau III).

- Les orangs-outans, les chimpanzés et les hommes qui sont caractérisés par des clavicules possédant simultanément une courbure externe 
Pan paniscus

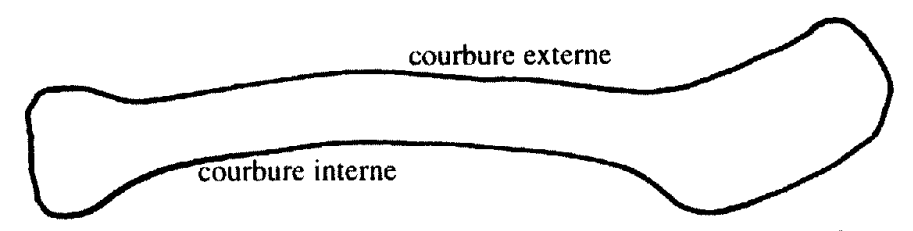

Pan troglodytes

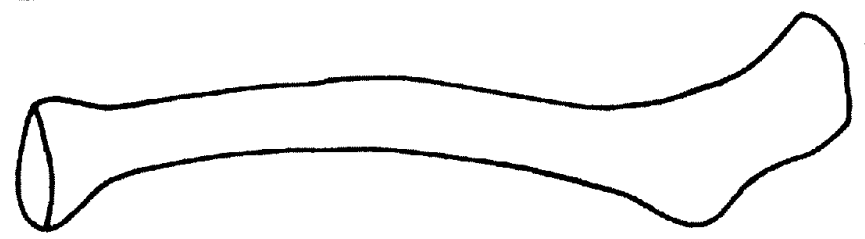

Homo sapiens sapiens

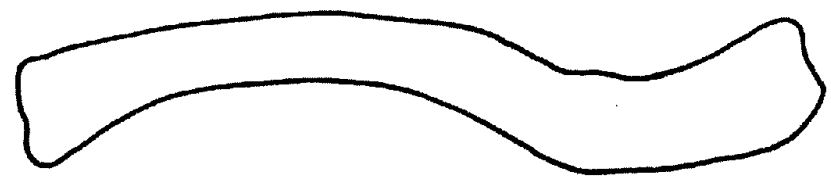

Pongo pygmaeus
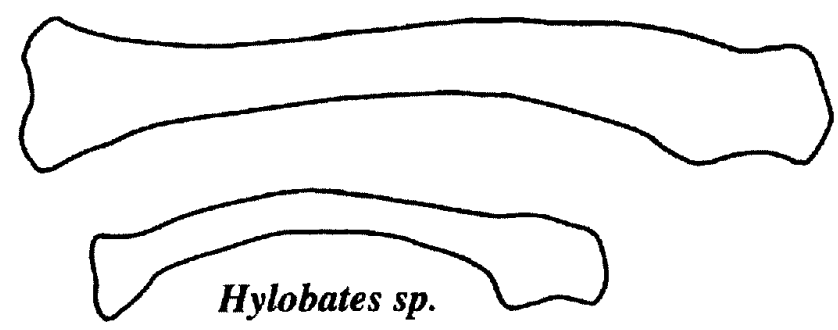

Papio hamadryas
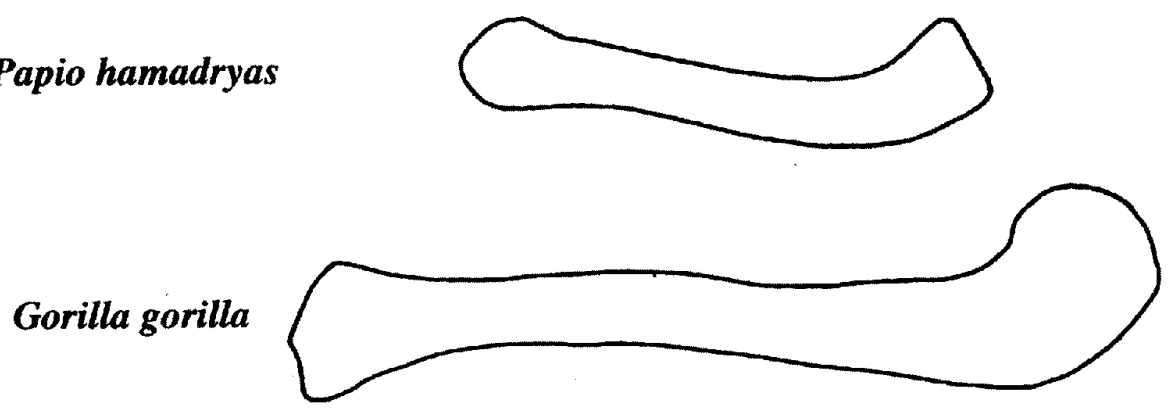

Figure 2. Clavicules droites en vue supérieure (les échelles ne sont pas respectées).

Figure 2. Right clavicles in superior view (scales are not respected).

et une interne (figure 3 et tableau III) qui sont en outre corrélées entre elles (tableau $I V$ ). Ces clavicules ne sont pas pour autant identiques. En effet, les clavicules des orangs-outans présentent les courbures les moins marquées, ce qui leurs confèrent un aspect presque rectiligne. Au contraire, les courbures sont beaucoup plus marquées chez les hommes et les chimpanzés, bien qu'il existe des dissemblances entre ces deux groupes. Autant la courbure externe ne présente pas de différence significative entre ces deux groupes (tableau $V$ ), autant la courbure interne est moins prononcée chez les chimpanzés que chez l'homme moderne (tableau $V$ ). La clavicule humaine est donc plus sinueuse que celle des chimpanzés contrairement à ce qui est classiquement décrit depuis Schultz (1930).

- Les gibbons sont caractérisés par des clavicules qui présentent une morphologie inversée par rapport à celle des gorilles et des babouins. En effet, les clavicules des gibbons présentent une courbure interne très prononcée contrairement à l'externe qui peut souvent manquer (tableau III et figure 3). 

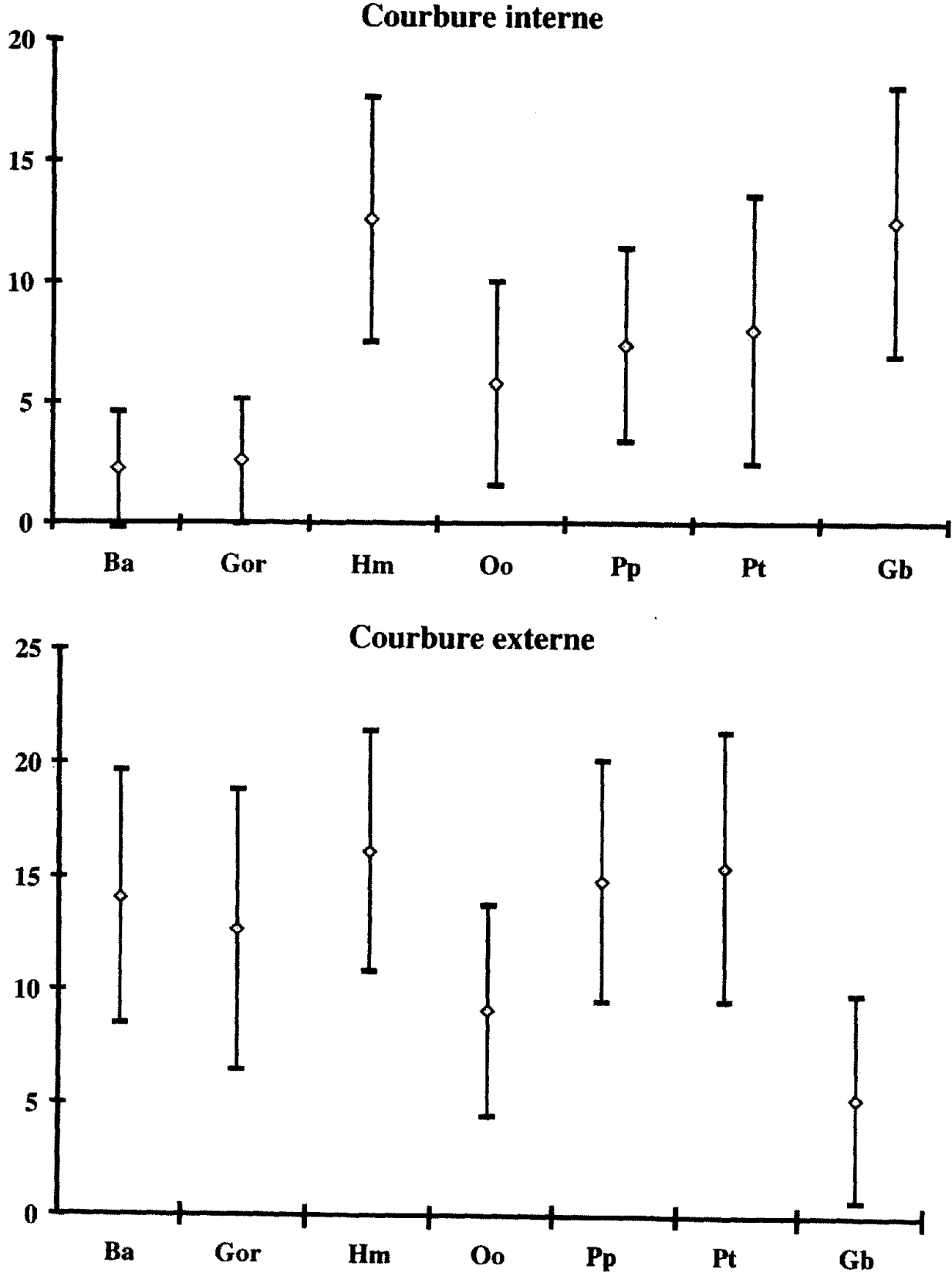

Figure 3. Valeurs moyennes et amplitudes de variation des courbures interne et externe chez les hominoïdes et les babouins.

Figure 3. Means and ranges of variation of the internal and external curves in hominoide and baboon.

\subsubsection{Les représentants fossiles du genre Homo}

En vue supérieure, les clavicules fossiles attribuées au genre Homo (figures 4 et 5) pré- sentent des courbures comprises dans l'intervalle de valeurs de l'homme moderne (tableau VI), et cela même si l'on ne tient compte que des individus complets (clavicules gauches de la Ferrassie I et de Kebara, clavicules droites de 
Tableau IV. Valeurs du coefficient de corrélation de Pearson $r$ entre les courbures externe et interne chez l'homme, les chimpanzés et les orangs-outans

Table IV. Pearson coefficient of correlation $r$ between the internal and the external curves in man, chimpanzees and orang-utan.

\begin{tabular}{lccc}
\hline Espèce & $\mathrm{r}$ & $\mathrm{r}^{2}$ & $\mathrm{p}$ \\
\hline Homo sapiens sapiens & 0,6 & 0,4 & 0,00 \\
Pan troglodytes & 0,8 & 0,7 & 0,00 \\
Pan paniscus & 0,6 & 0,3 & 0,01 \\
Orang-outan & 0,7 & 0,5 & 0,00 \\
\hline
\end{tabular}

Régourdou, d'ATD6-50 et de KNM-WT 15000 ). Cependant, ces clavicules fossiles ne présentent pas toutes une morphologie identique à celle de l'homme moderne en vue supérieure. En effet, KNM-WT 15000 (Homo ergaster) présente des valeurs très proches entre les courbures externe et interne. En d'autre termes, les deux courbures sont aussi marquées l'une que l'autre. Or, chez l'homme moderne la courbure externe est toujours plus prononcée que l'interne. Au contraire, la clavicule ATD6-50 (Homo antecessor) présente, en vue supérieure, une morphologie moderne avec une courbure externe plus prononcée que l'interne. Cependant, ces

Tableau V. Test-t entre les courbures interne et externe des clavicules de chimpanzé commun, de bonobo et d'homme moderne.

Table V. T-test between the internal and the external curves of common chimpanzee, pygmy chimpanze and man clavicles.

\begin{tabular}{|c|c|c|c|c|c|c|c|}
\hline \multirow[b]{2}{*}{ Group } & \multicolumn{3}{|c|}{ Courbure interne } & \multicolumn{4}{|c|}{ Courbure externe } \\
\hline & $\mathrm{n}$ & mean & sd & Group & $\mathrm{n}$ & mean & sd \\
\hline Pan troglodytes & 26 & 8,1 & 2,8 & Pan troglodytes & 26 & 15,4 & 3,0 \\
\hline Pan paniscus & 19 & 7,5 & 2,0 & Pan paniscus & 19 & 14,8 & 2,6 \\
\hline Separate variances & $\mathrm{T}=0,9$ & $\mathrm{DF}=43,0$ & Prob $=0,4$ & Separate variances & $\mathrm{T}=0,7$ & $\mathrm{DF}=41,2$ & Prob $=0,5$ \\
\hline Pooled variances & $\mathrm{T}=0,8$ & $\mathrm{DF}=43,0$ & Prob $=0,4$ & Pooled variances & $\mathrm{T}=0,7$ & $\mathrm{DF}=43,0$ & Prob $=0,5$ \\
\hline Group & $\mathrm{n}$ & mean & sd & Group & $\mathrm{n}$ & mean & sd \\
\hline Pan troglodytes & 26 & 8,1 & 2,8 & Pan troglodytes & 26 & 15,4 & 3,0 \\
\hline Homo sapiens sapiens & 33 & 12,6 & 2,5 & Homo sapiens sapiens & 33 & 16,1 & 2,6 \\
\hline Separate variances & $\mathrm{T}=-6,5$ & $\mathrm{DF}=51,1$ & Prob $=0,00$ & Separate variances & $\mathrm{T}=-0,9$ & $\mathrm{DF}=50,7$ & Prob $=0,4$ \\
\hline Pooled variances & $T=-6,5$ & $\mathrm{DF}=57,0$ & Prob $=0,00$ & Pooled variances & $\mathrm{T}=-0,9$ & $\mathrm{DF}=57,0$ & Prob $=0,4$ \\
\hline
\end{tabular}

Tableau VI. Valeurs des courbures externe et interne chez les hommes de Néandertal, Homo antecessor, Homo ergaster et Homo habilis.

Table VI. Values of the internal and external curves in Neandertal, Homo antecessor, Homo ergaster and Homo habilis.

\begin{tabular}{llcc}
\hline & Individu & Courbure interne & Courbure externe \\
\hline Néandertaliens & Régourdou droite & 10,0 & 14,5 \\
& Régourdou gauche & 11,9 & 13,2 \\
& Kebara gauche & 11,1 & 16,5 \\
Kebara droite & 11,3 & 9,5 \\
& La Ferrassie 1 droite & 13,2 & 14,2 \\
& La Ferrassie 1 gauche & 12,3 & 17,7 \\
& Krapina 4 gauche & 8,3 & 11,1 \\
& Neanderthal droite & 13,2 & 16,7 \\
& Moyenne & $\mathbf{1 1 , 4}$ & $\mathbf{1 4 , 2}$ \\
Homo ergaster & Ecart-type & 1,7 & $\mathbf{2 , 8}$ \\
KNM-WT 15 000 droite & 13,1 & 14,6 \\
Homo antecessor & KNM-WT 15 000 gauche & 15,3 & 14,7 \\
Homo habilis & Gran Dolina droite & 12,4 & 18,7 \\
\hline
\end{tabular}




\section{Néandertaliens}
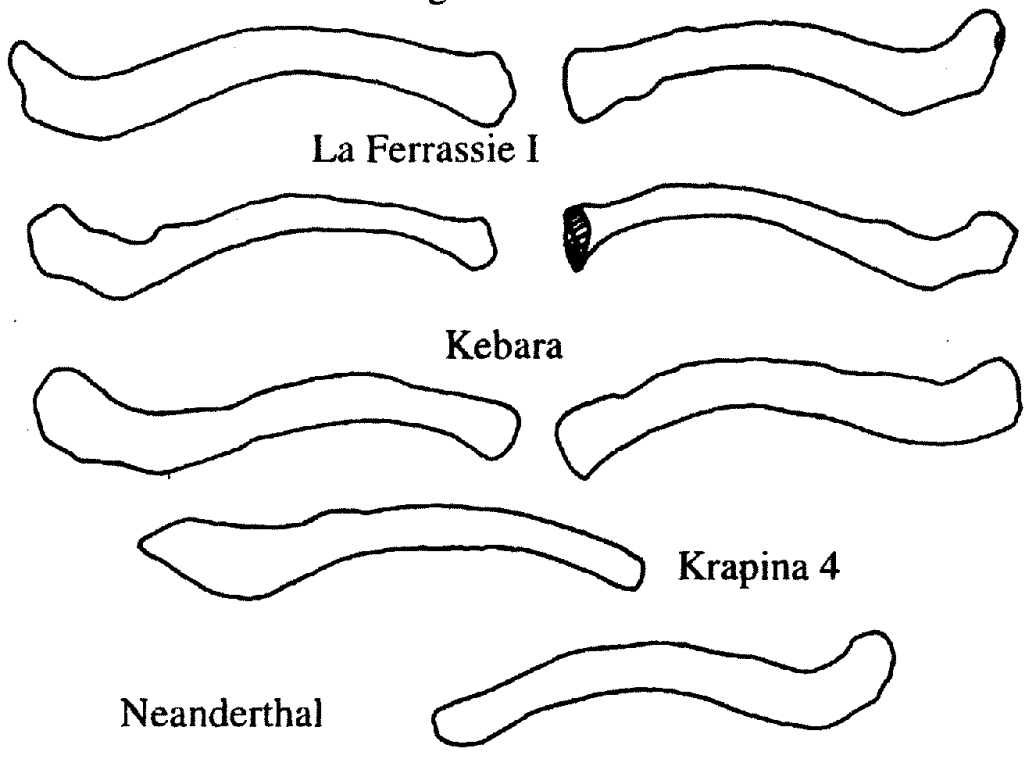

\section{Homo ergaster}

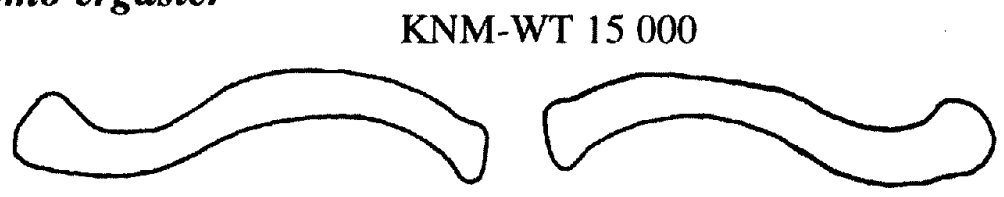

\section{Homo antecessor}
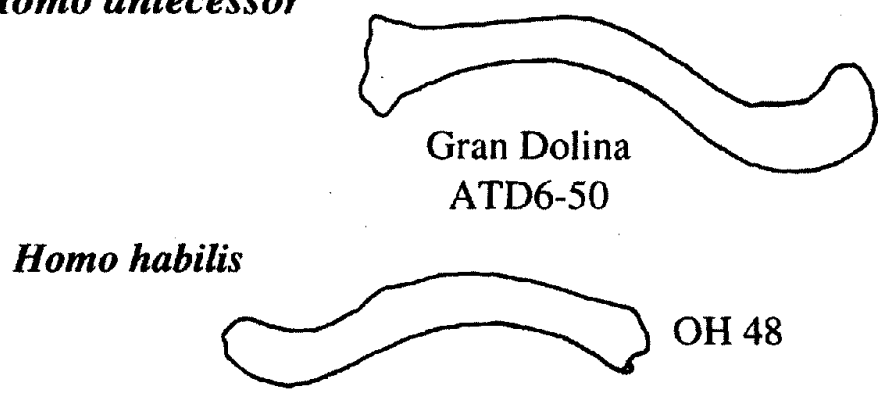

Figure 4. Clavicule de Néandertaliens, d'Homo antecessor, Homo ergaster, et Homo habilis en vue supérieure (les échelles ne sont pas respectées).

Figure 4. Neandertal, Homo antecessor, Homo ergaster and Homo habilis clavicles in superior view (scales are not respected).

valeurs ne seraient pas exceptionnelles chez l'homme moderne, contrairement aux affirmations de Carretero et al. (1999).

L'état fragmentaire de la clavicule $\mathrm{OH} 48$ (Homo habilis) fait que la courbure interne est plus prononcée que l'externe (tableau VI). En effet, l'extrémité sternale est pratiquement intacte alors qu'il manque une grande partie de l'extrémité acromiale (Napier, 1965). Ainsi, la courbure interne n'est que faiblement sous-estimée et se situe à la limite supérieure de l'intervalle de variation de l'homme moderne et pratiquement en dehors de celui du chimpanzé. Cet individu possédait donc des courbures très pro- 

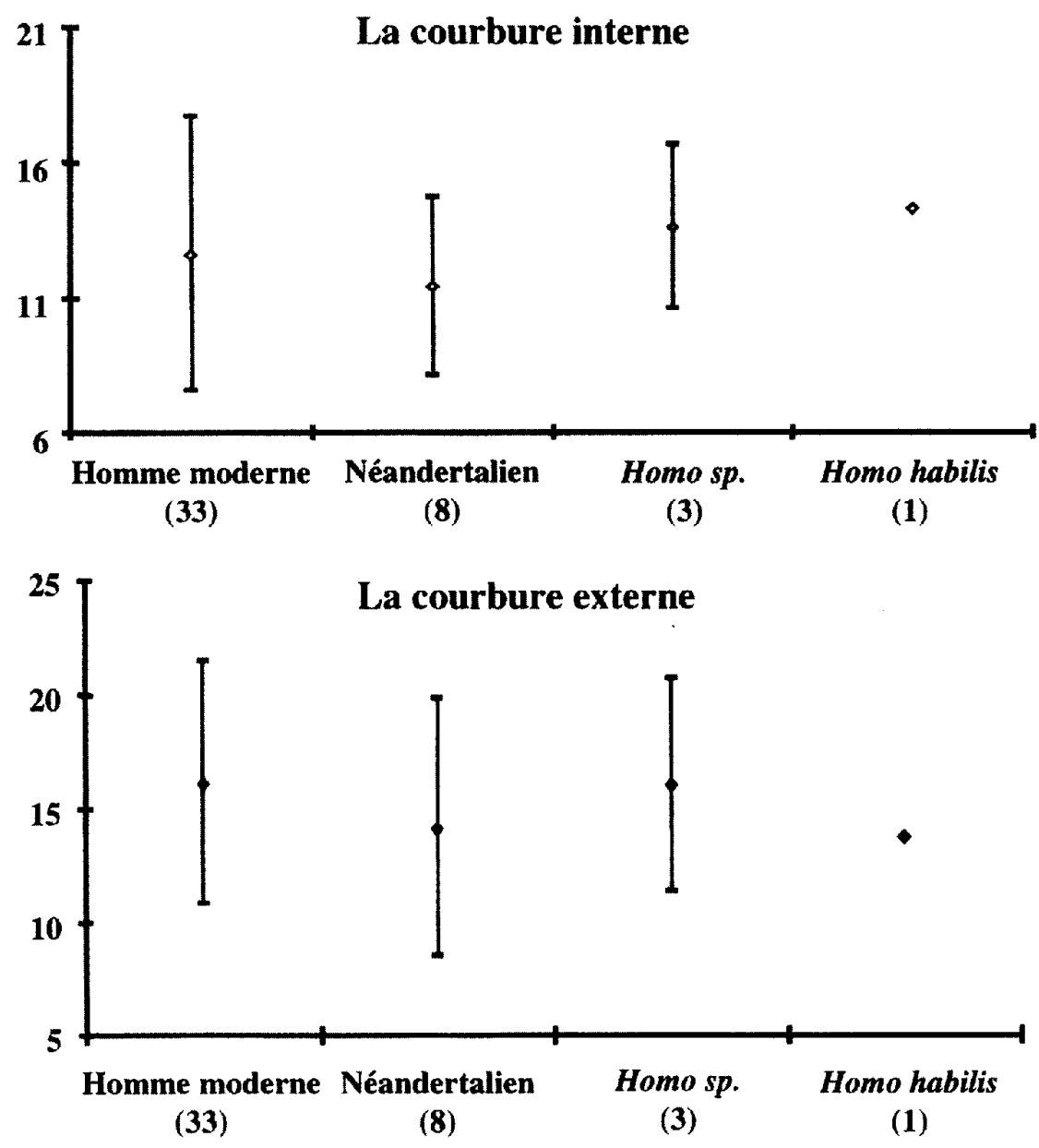

Figure 5. Valeurs moyennes et amplitudes de variation des courbures en vue supérieure chez l'homme moderne et diffërents groupes fossiles. Homo sp. regroupe Homo ergaster et Homo antecessor. Les chiffres entre parenthèse rappellent les effectifs.

Figure 5. Means and ranges of variation of clavicle curves in superior view in modern man and several human fossiles. Homo sp. means Homo ergaster plus Homo antecessor. Number in brackets indicates the number of specimens.

noncées en vue supérieure. Mais qu'en est-il exactement au sein de ce taxon ? Il faudrait plus de clavicules afin de conclure définitivement. que :

Ces résultats sont importants car ils montrent

- Les clavicules néandertaliennes ne sont pas plus sinueuses que celles de l'homme moderne contrairement à ce qui est fréquemment énoncé (Boule, 1912 ; Heim, 1974, 1982a, 1982b ; Vandermeersch et Trinkaus, 1995).

- Les clavicules d'Homo antecessor et d'Homo ergaster ne sont pas identiques. En effet, la morphologie claviculaire de KNM-WT 15000 n'est pas semblable à celle de l'homme moderne, comme le considèrent Walker et
Leakey (1993), contrairement à celle d'ATD650 qui s'en rapproche beaucoup plus.

- Les fortes courbures en vue supérieure sont apparues tôt dans l'histoire de l'humanité, sans pour autant être parfaitement identiques à la morphologie humaine moderne.

\subsection{Les courbures en vue postérieure}

\subsubsection{Les grands singes et l'homme moderne}

En vue postérieure les courbures claviculaires, constituant la déflexion (Matiegka, 1938), permettent de distinguer quatre groupes (figures ( et 7) : 

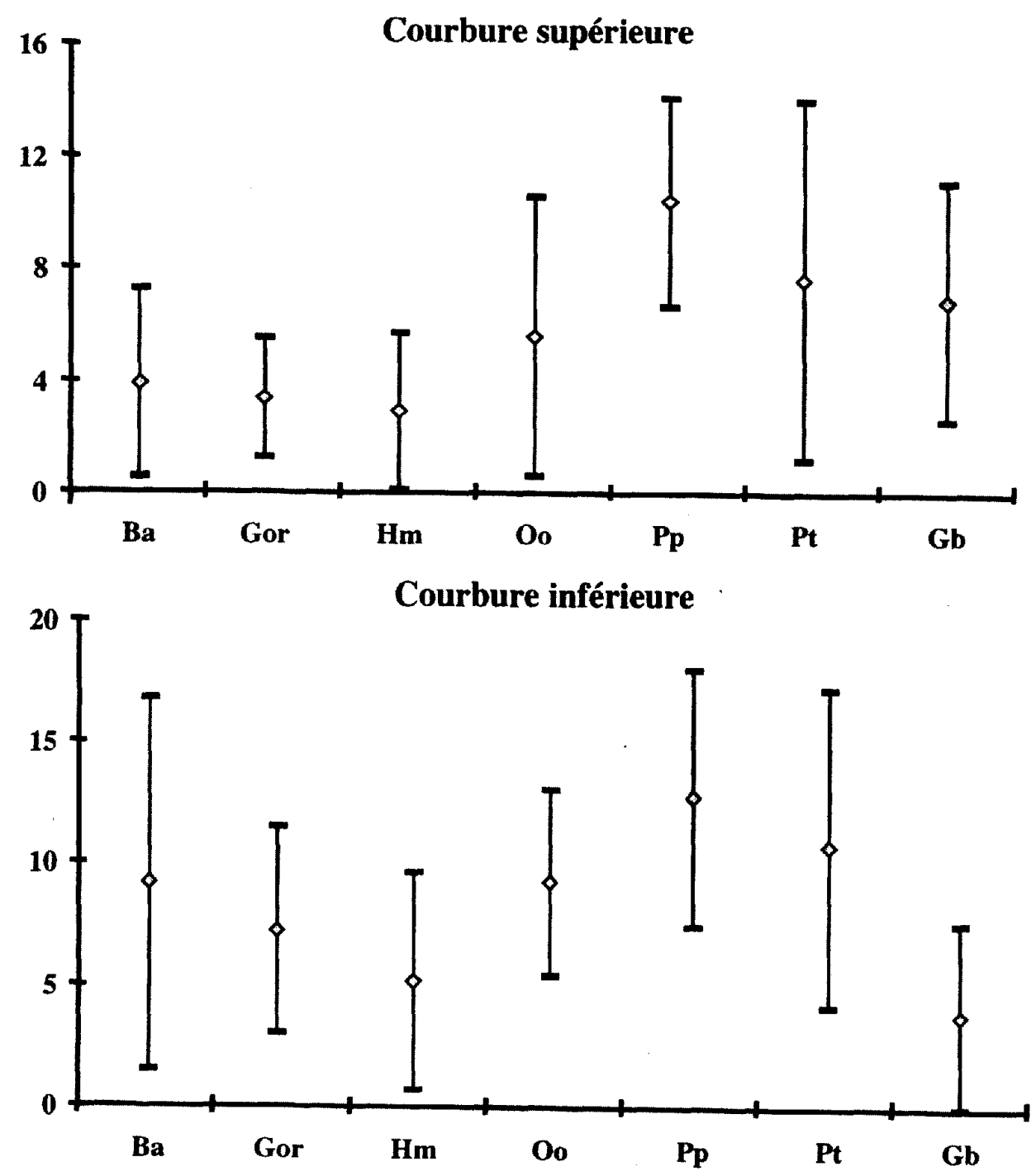

Figure 6. Valeurs moyennes et amplitudes de variation des courbures supérieure et inférieure chez les hominoïdes et les babouins.

Figure 6. Means and ranges of variation of the superior and inferior curves in hominoids and baboon.

- Les grands singes qui présentent toujours deux courbures en vue postérieure : une inférieure à l'extrémité latérale de la clavicule et une supérieure à l'extrémité médiale. Cés courbures sont plus ou moins développées selon les espèces, mais elles sont toujours présentes (tableau VII). Par ailleurs, la courbure inférieure est toujours plus prononcée que la supérieure pour une clavicule donnée (tableau VII).

- Les babouins qui présentent des clavicules dont la courbure inférieure est toujours présente alors que la supérieure est absente ou faiblement marquée.
- Les gibbons qui sont caractérisés par une seule courbure en vue postérieure, la courbure supérieure.

- L'homme moderne qui présente deux morphologies claviculaires distinctes. La première, et la plus fréquente $(84,8 \%)$, correspond à une clavicule ne présentant qu'une courbure inférieure, qui de surcroît n'est pas très prononcée. Ce sont les clavicules de type I (Olivier, 1951b). Par ailleurs, certaines clavicules $(24,2 \%)$ présentent en plus une courbure supérieure de 1'extrémité acromiale. Ce sont les clavicules de type III (Olivier, 1951b). La seconde morphologie, 
Tableau VII. Caractéristiques des courbures inférieure et supérieure des espèces actuelles étudiées ici.

Table VII. Characteristics of the clavicule curves in posterior view (inferior and superior).

\begin{tabular}{lcccccc}
\hline & \multicolumn{3}{c}{ Courbure infërieure } & \multicolumn{3}{c}{ Courbure supérieure } \\
\hline Espèce (nombre de pièces) & moyenne & écart-lype & variance & moyenne & écart type & variance \\
Homo sapiens sapiens (33) & 5,1 & 2,3 & 5,3 & 1,1 & 1,7 & 2,9 \\
Pan troglodytes (26) & 10,7 & 3,3 & 10,9 & 7,6 & 3,2 & 10,2 \\
Pan paniscus (19) & 12,7 & 3,0 & 9,0 & 10,4 & 1,9 & 3,6 \\
Gorilla gorilla (33) & 7,2 & 2,2 & 4,8 & 3,4 & 1,1 & 1,2 \\
Pongo pygmaeus (24) & 9,2 & 1,9 & 3,6 & 5,6 & 2,5 & 6,3 \\
Hylobates sp. (22) & 3,8 & 1,9 & 3,6 & 6,9 & 2,1 & 4,4 \\
Papio hamadryas (28) & 9,2 & 3,8 & 14,4 & 0,8 & 1,8 & 3,2 \\
\hline
\end{tabular}

Tableau VIII. Valeurs des courbures inférieure et supérieure chez les hommes de Néandertal, Homo antecessor, Homo ergaster et Homo habilis.

Table VIII. Values of the inferior and superior curves in Neandertal, Homo antecessor, Homo ergaster and Homo habilis.

\begin{tabular}{llcc}
\hline & Individu & Courbure inférieure & Courbure supérieure \\
\hline Néandertaliens & Régourdou droit & 7,4 & 8,0 \\
& Régourdou gauche & 3,0 & 3,2 \\
& Kebara gauche & 4,9 & 0,0 \\
& Kebara droite & 3,4 & 0,0 \\
& La Ferrassie 1 droite & 5,4 & 5,8 \\
& La Ferrassie 1 gauche & 8,2 & 2,6 \\
& Krapina 4 gauche & 3,2 & 2,9 \\
& Neanderthal droite & 7,4 & 6,2 \\
& Moyenne & $\mathbf{5 , 4}$ & $\mathbf{3 , 6}$ \\
Homo ergaster & Ecart-type & $\mathbf{2 , 1}$ & $\mathbf{2 , 9}$ \\
& KNM-WT 15 000 droite & 5,0 & 7,4 \\
Homo antecessor & KNM-WT 15 000 gauche & 5,3 & 8,1 \\
Homo habilis & Gran Dolina droite & 8,8 & 5,4 \\
\hline
\end{tabular}

peu fréquente $(15,2 \%)$, correspond à une clavicule ayant un morphotype grand singe. Ces clavicules, correspondant au type II, présentent des courbures supérieure et inférieure toujours faiblement développées par rapport à ce qui existe chez les grands singes (figure 8).

\subsubsection{Les hommes fossiles}

Les néandertaliens

A l'exception des clavicules de Kebara toutes les pièces néandertaliennes possèdent une courbure inférieure latérale et une courbure supérieure médiale (figure 9). Autrement dit, $75 \%$ des clavicules néandertaliennes étudiées présentent une morphologie de type II, alors que chez l'homme moderne cela représente $15 \%$ des clavicules.
Cependant, la morphologie des clavicules néandertaliennes, en vue postérieure, est particulière et ne correspond à aucun schéma actuel, ni à celui de l'homme moderne ni à celui des grands singes. En effet, la courbure supérieure chez l'homme moderne est nettement moins prononcée, quand elle existe, que chez les néandertaliens. L'amplitude et la moyenne de la courbure supérieure des clavicules néandertaliennes sont proches de celles des orangs-outans (figure 10 et tableaux VII et VIII) contrairement à l'inférieure qui est proche de celle de l'homme moderne (tableaux VII et VIII). En outre, certaines clavicules néandertaliennes (Régourdou droite et gauche ; la Ferrassie I droite) présentent une courbure supérieure plus prononcée que l'inférieure, ce qui ne se retrouve chez aucun autre primate. 

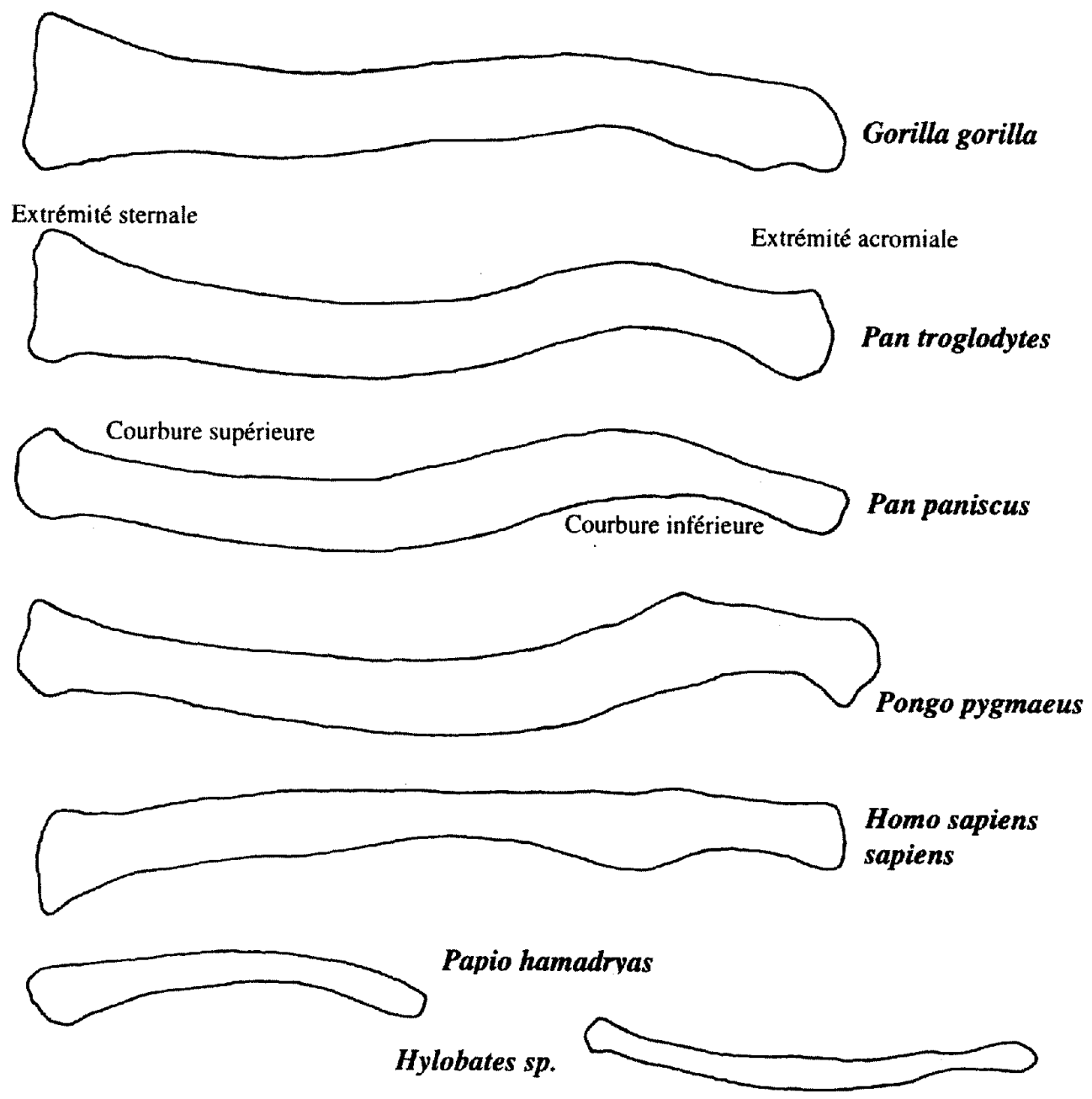

Figure 7. Clavicules droites en vue postérieure (les échelles ne sont pas respectées).

Figure 7. Right clavicles in posterior view (scales are not respected).

\section{Homo ergaster et Homo antecessor}

Les clavicules de Nariokotome (KNM-WT 15000 ) et d'Homo antecessor (ATD6-50) présentent une double courbure en vue postérieure (figure 11 et tableau VIII). Autant les valeurs des courbures inférieures des clavicules de KNM-WT 15000 et d'ATD6-50 sont comprises dans l'intervalle de valeurs de l'homme moderne (tableau VIII), autant les valeurs de la courbure supérieure de ces fossiles sont nettement au dessus de l'intervalle actuel (pour les clavicules modernes présentant cette courbure). De plus, les clavicules de Nariokotome ont une courbure supérieure plus prononcée que l'inférieure ce qui n'est pas le cas chez ATD6-50.
Ainsi, la morphologie claviculaire en vue postérieure des Homo erectus au sens large est différente de celle de l'homme moderne et se rapproche plutôt de celle des néandertaliens car :

- les deux courbures existent en vue supérieure et sont bien individualisées,

- la courbure supérieure peut être plus prononcée que l'inférieure, ce qui ne se retrouve que chez les néandertaliens.

\section{Homo habilis}

La clavicule d'OH 48, la seule clavicule d'Homo habilis connue, présente deux courbures en vue postérieure (figure 11 ), mais elles 
Extrémité sternale

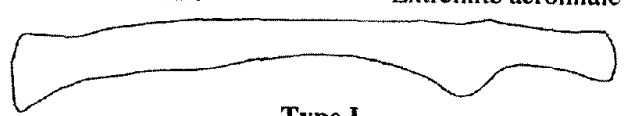

Type I

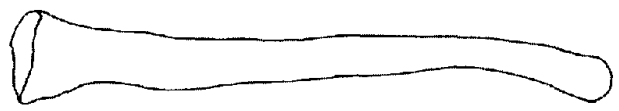

Type II

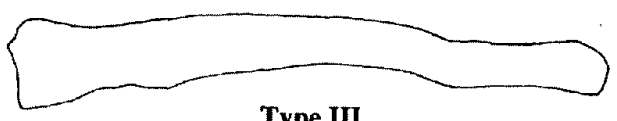

Type III

Figure 8. Les trois types de clavicules chez l'homme moderne.

Figure 8. The three types of modern man clavicle.

sont peu prononcées et entrent parfaitement dans l'intervalle de valeurs de l'homme moderne (tableau VIII). L'inférieure est la plus prononcée. Il est délicat d'affirmer que cette morphologie caractérise les Homo habilis car cette clavicule n'est pas complète (Napier, 1965). Cependant, cette déflexion avec une double courbure est plus fréquente chez les néandertaliens, Homo ergaster et Homo antecessor que chez l'homme moderne. Ainsi, la morphologie observée sur la clavicule $\mathrm{OH} 48$ devrait être proche de celle des Homo habilis en général.

\section{Discussion}

\subsection{Les courbures en vue supérieure}

Le muscle grand pectoral a un rôle primordial lors des mouvements de flexion chez l'homme, notamment lors de leur initiation (Gagey, 1985). L'efficacité de ce muscle chez l'homme est due au développement de la courbure interne de la clavicule qui crée un effet de manivelle et ainsi aide à l'élévation du bras en facilitant le pivotement de la scapula pour orienter la cavité glénoïdale vers le haut (Voisin, 2000b). Chez les primates peu d'espèces présentent une insertion claviculaire du grand pectoral étendue. Ces espèces sont 1'homme, les chimpanzés, les gorilles et les gibbons (Asthon et Oxnard, 1963). $\mathrm{Au}$ contraire, chez les orangs-outans cette insertion est très peu étendue et manque même fréquemment (Jouffroy, 1962 ; Sullivan et Osgood, 1927).

Les espèces présentant un développement important de l'insertion claviculaire du grand pectoral, ainsi qu'une courbure claviculaire interne marquée, sont les deux espèces de chimpanzés, l'homme et les gibbons. Le développement de la courbure interne est donc associé à une flexion puissante et rapide. Une telle disposition permet de développer la brachiation et / ou la manipulation.

Quelle que soit l'espèce d'Hominidae fossile, toutes les clavicules étudiées dans ce travail

\section{Extrémité acromiale Extrémité sternale Extrémité acromiale}
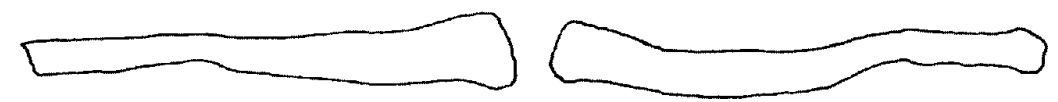

Régourdou
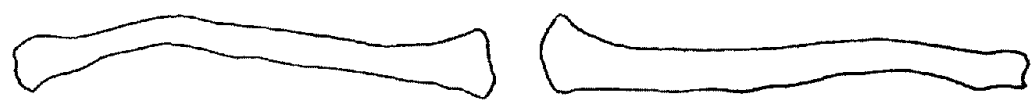

La Ferrassie I
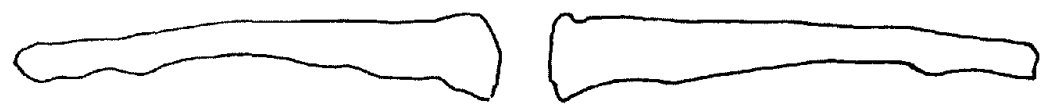

Kebara

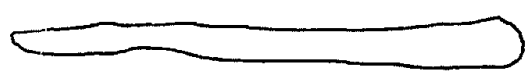

\section{Krapina 4}

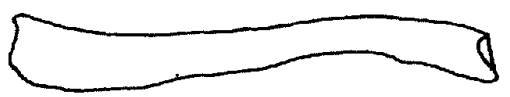

Neanderthal

Figure 9. Clavicules néandertaliennes en vue postérieure (les échelles ne sont pas respectées).

Figure 9. Neandertal clavicles in posterior view (scales are not respected). 

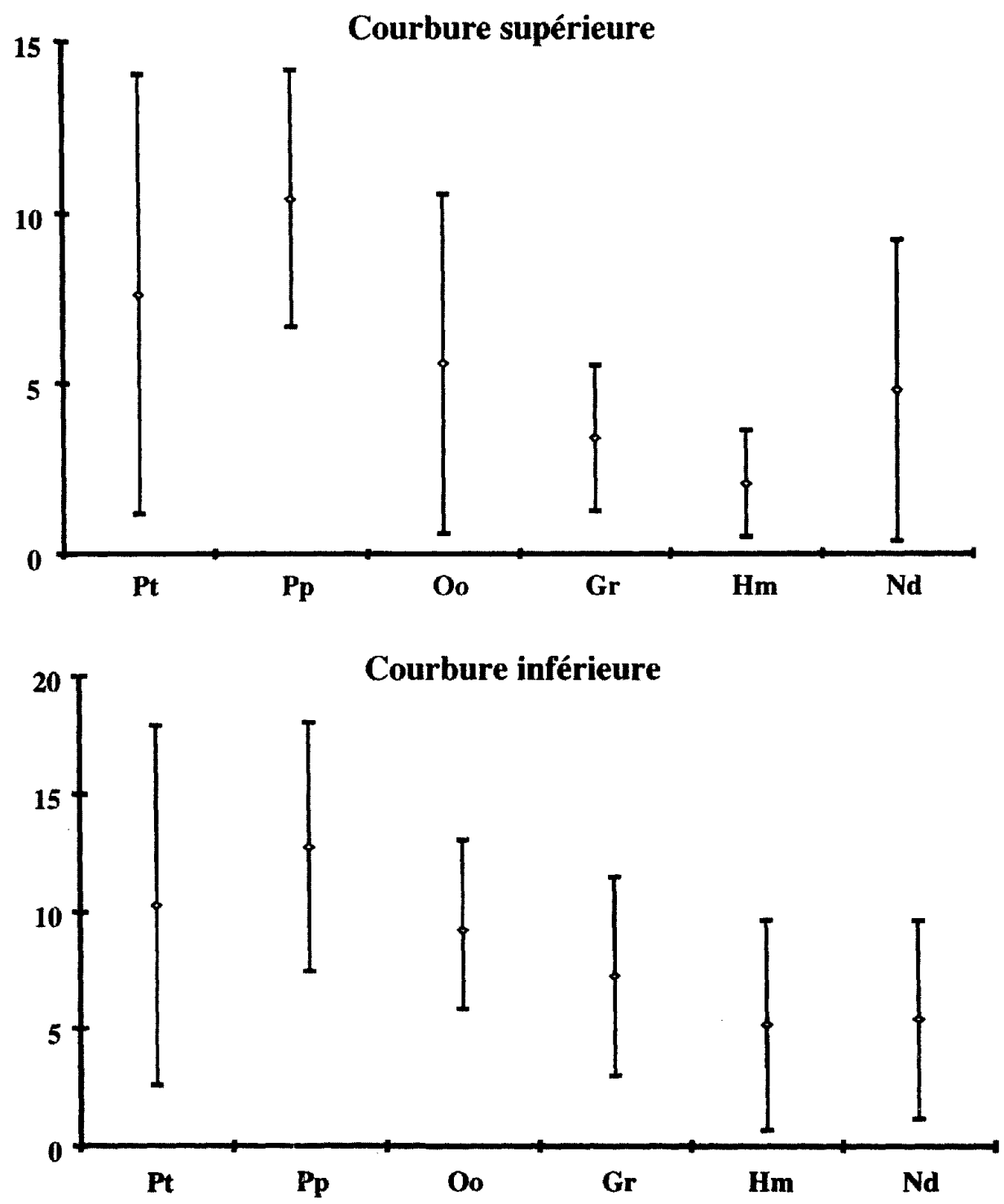

Figure 10. Comparaison des valeurs moyennes et des amplitudes de variation entre les Néandertaliens, les grands singes et l'homme moderne pour les courbures en vue postérieure.

Figure 10. Comparaison between means and ranges of variation of the Neandertal, great apes and modern man for the curves in posterior view.

présentent des courbures en vue supérieure identiques ou proches de celles de l'homme moderne (figure 5). Ainsi, la forte sinuosité décrite pour les clavicules néandertaliennes n'est pas une réalité physique, mais une impression due à leur extrême longueur. Bien que la clavicule $\mathrm{OH} 48$ (Homo habilis) ne soit pas complète (Napier, 1965) les courbures devaient être proches de celles de l'homme moderne, notamment la courbure interne. Les capacités fonctionnelles d'élévation du bras des espèces humaines fossiles étaient donc identiques à celles de l'homme moderne. De plus, la morphologie claviculaire humaine en vue supérieure est une adaptation à la bipédie car elle permet le maintient mécanique de l'épaule en position érigée, c'est-à-dire avec un coût énergétique minimum (Voisin, 2000b). La morphologie claviculaire humaine moderne en vue supérieure est donc apparue rapidement dans l'histoire de 


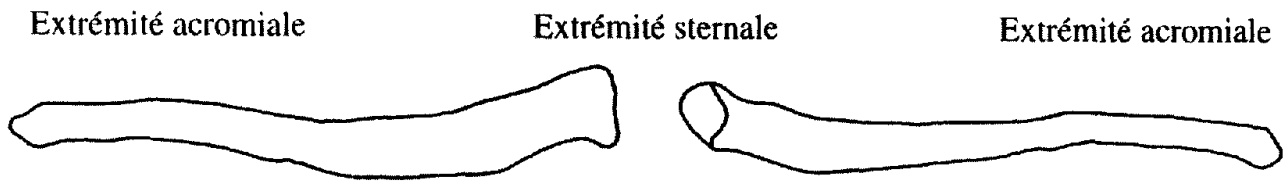

KNM-WT 15000 ; Homo ergaster
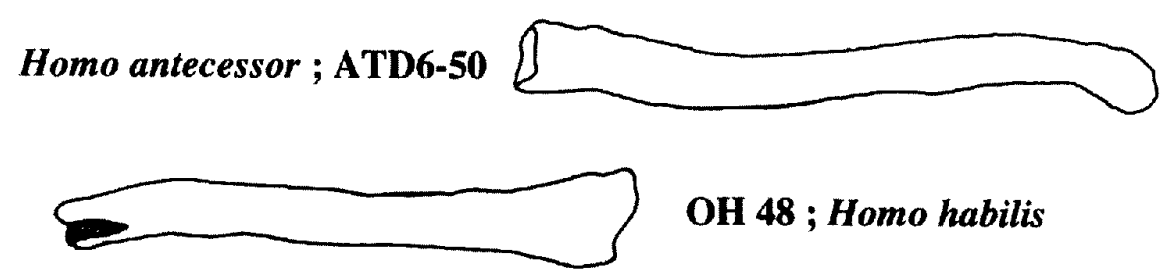

OH 48 ; Homo habilis

Figure 11. Clavicules d'Homo antecessor, Homo ergaster, et Homo habilis en vue postérieure (les échelles ne sont pas respectées).

Figure 11. Homo antecessor, Homo ergaster and Homo habilis clavicles in posterior view (scales are not respected).

l'homme, dès que la bipédie est devenue prépondérante et que la main s'est libérée des contraintes locomotrices.

Il existe peu de clavicules a peu près complètes attribuées aux Homo erectus. En dehors de celles étudiées dans ce travail, il existe deux autres restes, l'un africain et l'autre provenant de l'Inde. La clavicule africaine KNM-ER 1808 présente une morphologie et une sinuosité semblables à celles de l'homme moderne (Leakey et Walker, 1985). Au contraire, le reste découvert dans la vallée de la Narmada (Inde) et décrit comme une clavicule d'Homo erectus (Sankhyan, 1997), présente une morphologie particulière. En effet, les courbures sont très inégales (la méthode de mesure étant différente de la nôtre, toute comparaison métrique est impossible) et traduisent une forme plus proche de celle du gorille que de celle de l'homme moderne. Par ailleurs, cette clavicule est très courte, et ne rentre pas dans l'intervalle de valeurs actuelles (Voisin, 2000a). La morphologie particulière de la clavicule de la Narmada lui confère des fonctions plus proches de celle du gorille que de celle de l'homme moderne. Ainsi, cette clavicule ne semble pas appartenir au genre Homo.

\subsection{Les courbures en vue postérieure}

La clavicule travaille essentiellement en compression, transmettant la charge des membres supérieurs au squelette axial par l'intermédiaire de son grand axe (Jenkins, 1974 ; Fleagle,
1978 ; Mays et al,, 1999). Ainsi la morphologie claviculaire influence sur la diffusion des forces et donc sur les modes de déplacement de l'individu.

La brachiation correspond à un déplacement suspendu par les membres supérieurs (figure 12), associé à un balancement alterné de ces derniers afin de saisir un nouveau support (Fleagle, 1974).

Afin que la brachiation soit efficace et peu coûteuse en énergie, certaines contraintes sont nécessaires. En particulier, le centre d'inertie de l'individu doit toujours rester dans un plan vertical passant par le centre de rotation du pendule (Fleagle, 1974). Pour contrôler ce facteur essentiel, l'individu peut uniquement jouer sur les articulations du poignet, du coude et de l'épaule.

Lorsque le bras arrière de l'animal lâche le support, le corps réalise un double mouvement (figure 12) :

- Un mouvement de pendule.

- Une rotation du corps sous le bras d'appui qui place le thorax dans le sens du mouvement du pendule.

Lors de la brachiation, la présence de la clavicule permet de réaliser le mouvement de rotation du thorax sous la main d'appui car, en maintenant constant la distance acromio-manubrium, elle empêche la scapula de s'écraser sur le thorax. De plus, l'efficacité du mouvement de pendule lors de ce mode de déplacement dépend 


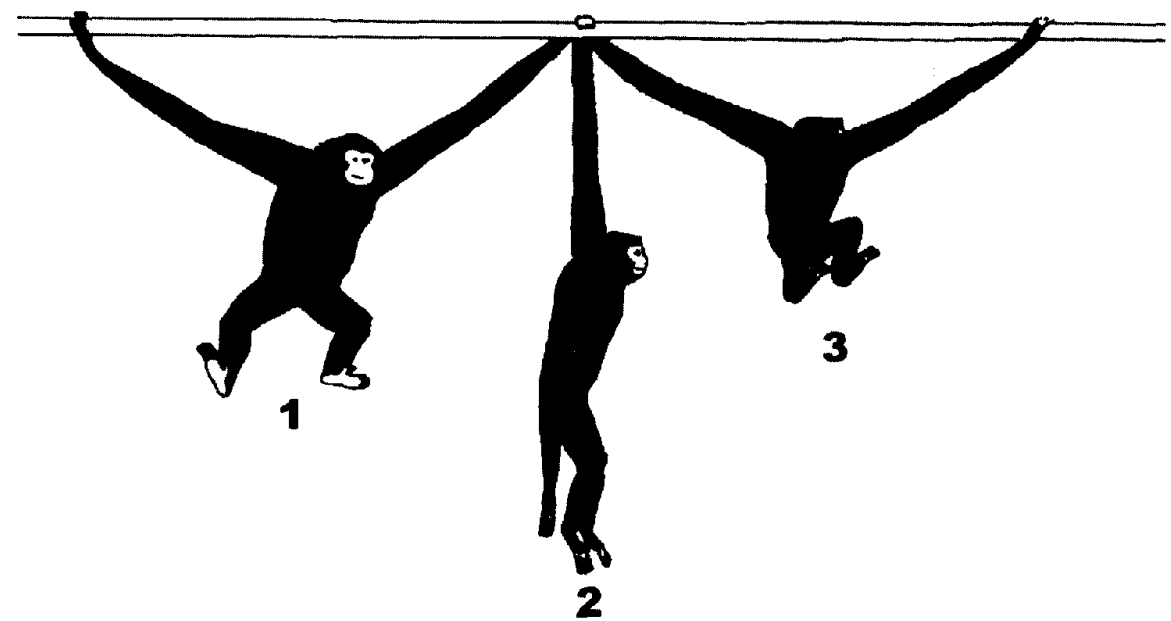

Figure 12. La brachiation chez le gibbon, la phase 1 correspond au départ du mouvement, la phase 3 à la fin du mouvement (d'après Fleagle, 1974).

Figure 12. Brachiation in the gibbon. Phase 1 is the start and phase 3 the end of the movement (redraw from Fleagle, 1974).

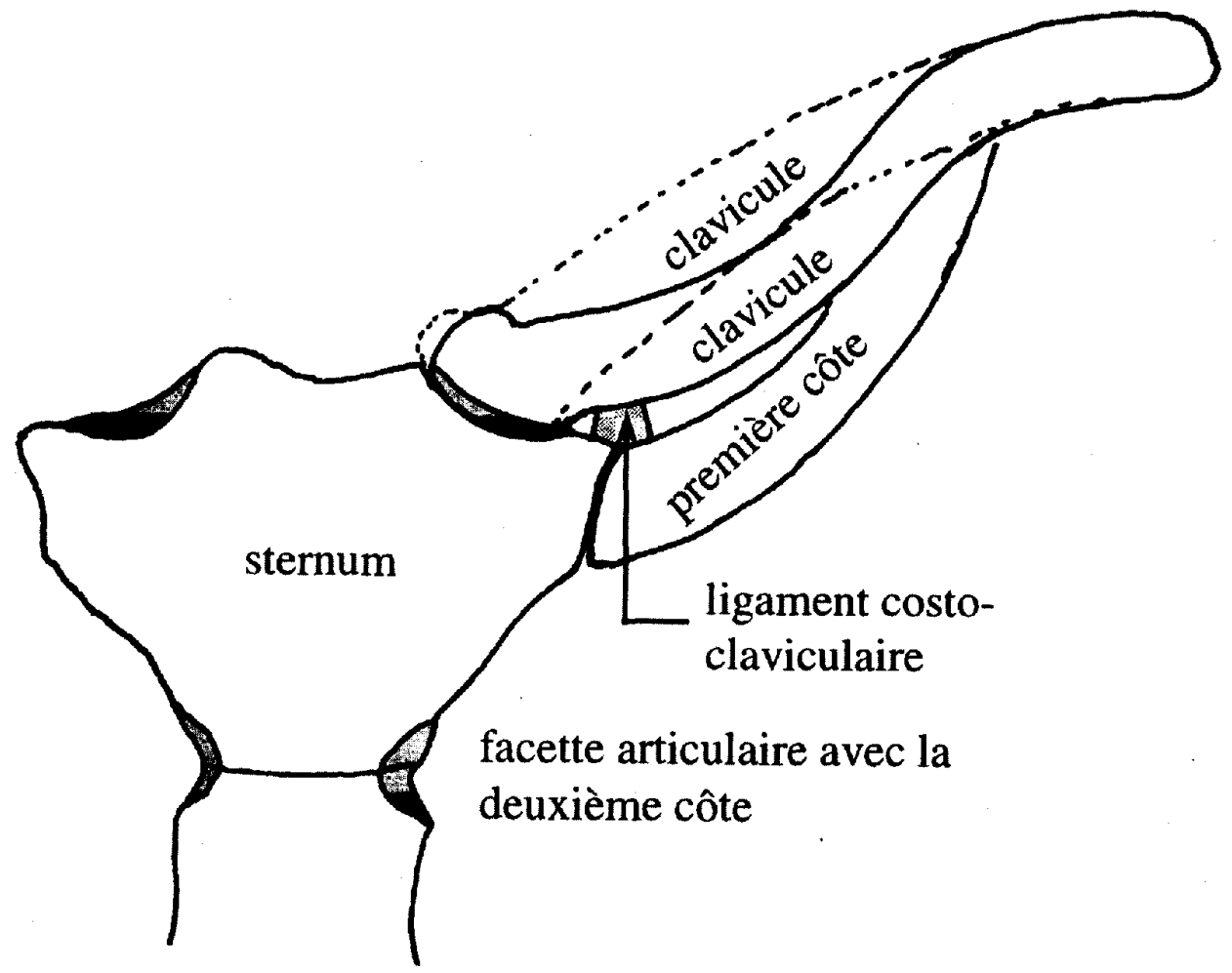

Figure 13. Clavicules associées à une scapula haute par rapport au thorax. En pointillés clavicule humaine (courbure inférieure unique). En traits pleins clavicule de grand singe (deux courbures). Noter la différence de hauteur par rapport au manubrium qui existe entre ces deux morphologies claviculaires (d'après Voisin, 2000c).

Figure 13. Clavicles associated with a high scapula in regard to the thorax. Dotted line: modern human clavicle (with a unique inferior curve), in full line the great apes clavicle (with two curves in posterior view). Note the difference in height in regard to the manubrium between the two clavicular morphology (from Voisin 2000c). 


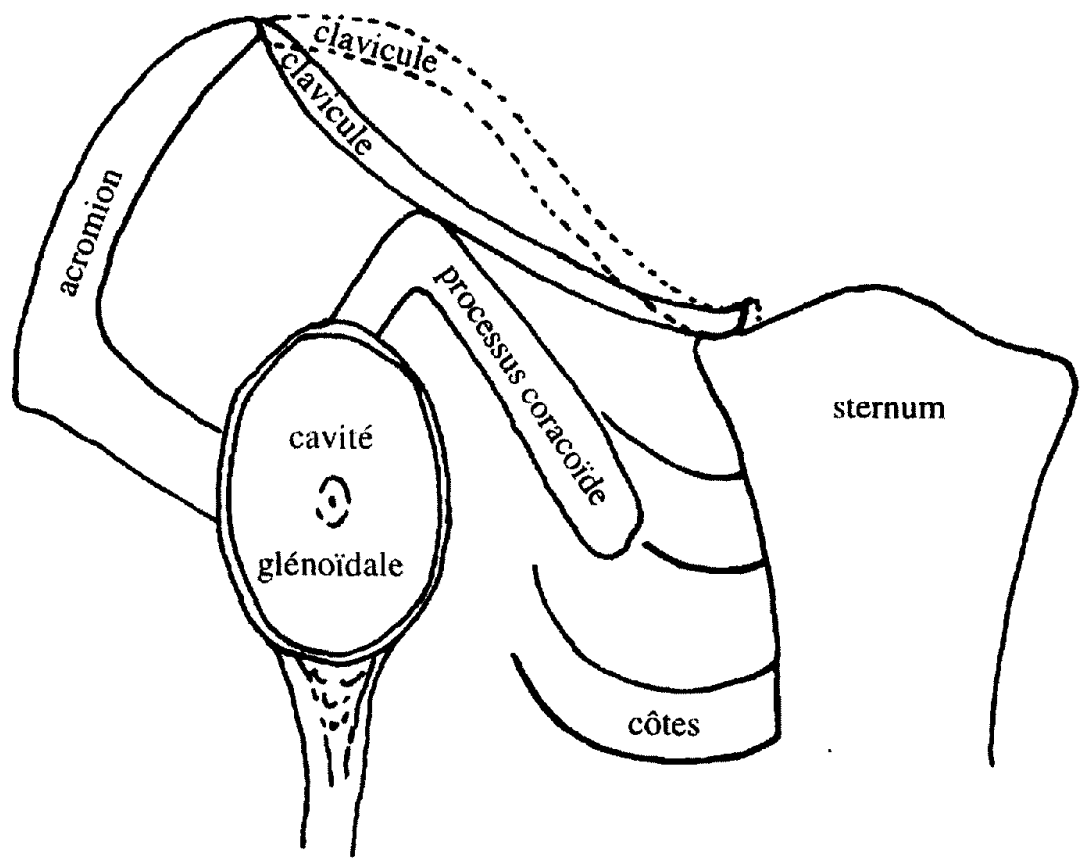

Figure 14. Relations entre la scapula, la clavicule et le sternum pour une clavicule de type gibbon (trait plein) et de type grand singe (trait pointillé) (d'après Voisin, 2000a).

Figure 14. Relations between scapula, clavicle and sternum for a gibbon clavicle (full line) and a great ape one (dotted line) (from Voisin, 2000a).

de la morphologie claviculaire en vue postérieure. Chez le gibbon, le mouvement de pendule est optimisé grâce à deux particularités claviculaires :

- La présence d'une courbure supérieure prononcée qui permet à l'extrémité sternale de la clavicule de rester parallèle au manubrium associée à une scapula haute par rapport au thorax (figure 13). Ainsi, le ligament costo-claviculaire et le muscle subclavier conservent leur fonction (Voisin, 2000a, 2000c) car ils ne subissent aucune élongation. Le ligament costo-claviculaire limite les mouvements verticaux et horizontaux de l'extrémité sternale de la clavicule (Kapandji, 1994). Un allongement de ce ligament augmenterait la mobilité de cette extrémité qui devrait alors être compensée par l'action de différents muscles tel que le subclavier. Cela entraînerait un coût énergétique plus élevé du mouvement, ainsi qu'un risque important de luxation de l'articulation sterno-claviculaire. Par ailleurs, chez les atèles, le muscle subclavier est très peu sollicité lors de la brachiation, alors qu'il l'est fortement lors du grimper sur structure verticale (Konstant et al., 1982), ce qui doit aussi être transposable aux Hylobatidae.

- L'absence de courbure inférieure, présente chez tous les autres primates, entraine nécessairement une articulation entre le processus coracoïde et la clavicule (figure 14). Cette articulation, bien qu'étant la conséquence du développement particulier des courbures en vue postérieure, accroît la rigidité de l'ensemble clavicule/scapula en augmentant la cohésion entre ces deux os. En effet, chez les autres primates un ou deux ligaments réalisent cette liaison. L'ensemble clavicule/scapula est alors moins rigide. La forte liaison entre ces os chez les gibbons améliore la diffusion des forces et surtout permet de limiter les mouvements du centre d'inertie de l'individu en dehors du plan vertical passant par la main d'appui.

Chez les primates quadrupèdes, la présence de la clavicule permet aux membres supérieurs de réaliser des mouvements en dehors du plan parasagittal, mais elle ne doit pas non plus entraver les déplacements quadrupèdes. La présence d'une courbure inférieure prononcée permet à la scapula de réaliser des mouvements de bascule 
importants lors de la marche, sans qu'elle aille buter contre la clavicule. Cependant, la faible cohésion de l'ensemble scapula / clavicule limite les capacités de brachiation et l'absence de courbure inférieure limite la remontée de la scapula le long du thorax lors des phases de suspension.

Chez les grands singes, la clavicule présente une déflexion caractéristique avec deux courbures. Ces espèces, caractérisées par une scapula haute et dorsale par rapport au thorax (Martin et O'Brien, 1939 ; Schultz, 1950 ; Sakka, 1985), pratiquent à la fois les déplacements suspendus et, au sol, le "knuckle-walking" (gorille \& chimpanzé) ou le "fist-walking" (orang-outan) (Asthon \& Oxnard, 1964). La morphologie claviculaire de ces primates, en vue postérieure, permet de répondre aux contraintes opposées que la locomotion suspendue et quadrupède imposent grâce à :

- L'existence d'une courbure supérieure médiale qui permet, nous l'avons vu chez le gibbon, de présenter une surface claviculaire à peu près parallèle au manubrium tout en associant une scapula haute par rapport au thorax.

- La courbure inférieure qui est toujours bien développée car elle est nécessaire au bon fonctionnement de l'épaule lors des déplacements quadrupèdes.

L'homme est caractérisé par l'émergence "cervico-céphalique" (Sakka, 1985), en regard des grands singes, car le cou se développe en hauteur entrainant la sortie de la tête hors des épaules. Selon Sakka (1985), ce phénomène est associé à une descente de la ceinture scapulaire le long du thorax. Par ailleurs, la clavicule est toujours orientée plus cranialement chez les grands singes que chez l'homme (Olivier, 1965 ; Sakka, 1985) et chez l'orang-outan elle réalise un angle de 30 à $50^{\circ}$ par rapport au plan transversal (Vallois, 1928). La descente de la ceinture scapulaire chez l'homme, en regard de celle des grands singes, expliquerait la présence d'une unique courbure inférieure. En effet, une double courbure prononcée en vue postérieure n'est pas compatible avec une scapula basse par rapport au thorax, car une telle disposition entraînerait une dislocation de l'articulation sterno-claviculaire.

En dehors des clavicules de Kebara, toutes les clavicules néandertaliennes étudiées dans le cadre de ce travail présentent une double courbure dans le plan frontal. Les valeurs de ces courbures sont relativement importantes et leurs proportions relatives ne correspondent à aucun primate actuel. Ces particularités montrent une architecture du complexe scapulaire chez les néandertaliens différente de celle caractérisant les hommes modernes. En effet, la double courbure en vue postérieure montre que la scapula est plus haute par rapport au thorax chez les néandertaliens que chez l'homme actuel. Cette disposition entraîne, chez les néandertaliens, un cou court en comparaison du nôtre (Spoor \& Wood, 1999 ; Voisin, 2000a, 2000c), bien que la hauteur de la colonne cervicale soit pratiquement identique à la nôtre (Heim, 1974, 1976 ; Vandermeersch, 1981). Ces différences d'architectures pourrait refléter la moindre "émergence cervico-céphalique" (Sakka, 1985) des néandertaliens en regard de l'homme moderne. En effet, chez les premiers, les insertions musculaires crâniennes sont plus hautes que chez l'homme moderne. Par ailleurs, ces différences d'architectures scapulaires entre ces deux groupes humains pourraient refléter des dissemblances fonctionnelles de certains muscles, notamment du trapèze. En effet, la partie crâniale de ce muscle est identique du point de vue anatomique entre l'homme et le chimpanzé, contrairement à sa fonction (Larson et al., 1991) et aux morphologies claviculaires en vue postérieure. Ainsi, la morphologie particulière de la clavicule néandertalienne ne refléterait-elle pas des fonctions différentes du trapèze par rapport à l'homme moderne ? La morphologie des canaux semi-circulaires des néandertaliens présente un argument dans ce sens puisqu'elle semble indiquer que le contrôle de l'équilibre ne nécessitait pas exactement les mêmes mouvements crâniens que chez l'homme moderne (Spoor \& Wood, 1999).

Par ailleurs, cette position plus haute de la scapula néandertalienne par rapport au thorax entraîne une clavicule qui n'est pas orientée presque horizontalement comme chez l'homme moderne mais plus oblique. Or, pour un diamètre identique du thorax, plus la scapula est haute, plus la clavicule doit s'allonger. Ainsi, la très grande longueur des clavicules néandertaliennes ne serait due ni à une largeur d'épaule exceptionnelle comme cela est classiquement admis (Patte, 1955; Heim, 1974, 1982a ; Vandermeersch et Trinkaus, 1995), ni au développement important des insertions claviculaires du muscle sterno-cleido-mastoïdien et du trapèze (Nara, 1994). Quoi qu'il en soit, la très 
grande longueur des clavicules néandertaliennes pourrait traduire une capacité au jet plus grande chez ces derniers que chez l'homme moderne (Voisin 2000a).

Les clavicules d'ATD6-50, de KNM-WT 15000 et d'OH 8 montrent la présence d'une double courbure en vue postérieure. Ainsi, ces individus devaient présenter une scapula haute par rapport au thorax, tout comme les néandertaliens. Cette architecture de l'épaule serait alors habituelle dans le genre Homo. Au contraire, celle de l'homme moderne, caractérisée par une scapula beaucoup plus basse par rapport thorax, serait une particularité dans l'histoire de notre genre. En d'autres termes, l'architecture de l'épaule néandertalienne serait héritée d'une morphologie ancestrale, contrairement à celle de l'homme moderne qui est une innovation. L'architecture de la ceinture scapulaire permet donc de définir deux groupes d'humains, qui peuvent aussi être déterminés d'après la morphologie de la ceinture pelvienne (Marchal, 2000a, 2000b).

Walker et Leakey (1993) considèrent que les clavicules de KNM-WT 15000 ne présentent que la courbure inférieure. Cette divergence d'interprétation s'explique par la morphologie particulière de cette clavicule qui rend difficile la détermination de la face inférieure par rapport au bord postérieur. La méthodologie employée dans cette étude lève cette difficulté.

\section{Conclusion}

Ce premier travail montre que l'étude de la clavicule est très riche en informations, et que cet os présente un intérêt particulier en anthropologie et paléoanthropologie. En vue supérieure sa morphologie donne des indications sur les capacités d'élévation du bras, alors qu'en vue postérieure elle participe à la description de l'architecture de l'épaule. Elle informe sur la position de la scapula par rapport au thorax (latérale ou dorsale, haute ou basse).

Par ailleurs, cette étude a permis de montrer que la scapula est plus haute par rapport au thorax chez Homo habilis, Homo ergaster, Homo antecessor et les néandertaliens que chez l'homme moderne. Cette descente du système scapulaire chez l'homme moderne est-il à mettre en relation avec le désenclavement musculaire de la tête ? En effet, au cours de l'évolution les insertions musculaires descendent de plus en plus bas le long du crâne, notamment le trapèze qui s'insère sur la base du crâne et la ceinture scapulaire.

Les courbures en vue supérieure, au contraire, ne montrent pas de différences (seules celles de KNM-WT 15000 ne sont pas exactement identiques aux clavicules des hommes modernes) avec l'homme moderne. Ce résultat est important car il contredit les affirmations précédentes qui considéraient, notamment, que les clavicules néandertaliennes étaient plus sinueuses que les nôtres. Cette impression est due à la grande longueur des clavicules néandertaliennes.

\section{Remerciement}

Je tiens à remercier tout particulièrement Monsieur le Professeur Henry de Lumley qui m'a accepté au sein du Laboratoire de Préhistoire du Muséum National d'Histoire Naturelle ainsi qu'à Madame Marie-Antoinette de Lumley qui suit l'avancement de mes travaux. Mes remerciements vont aussi aux professeurs André Langaney, Daniel Robineau, Wim Van Neer, Paula Jenkins et Chris Stringer qui m'ont autorisé à consulter respectivement les collections des Laboratoires d'Anthropologie Biologique du Musée de l'Homme (Paris, France), d'Anatomie Comparée du Muséum National d'Histoire Naturelle (Paris, France), du Musée Royal d'Afrique Centrale (Tervuren, Belgique) et du Natural History Museum, Zoological Group et Paleontological Group (Londres, Grande Bretagne). Je remercie aussi toutes les personnes de l'Institut de Paléontologie Humaine qui m'ont apporté leur soutien.

\section{Références}

Apostolakis, G., 1934. La clavicule de l'Homme. Arch. Anat. Histol. Embryol. 18, 169-180.

Ashton, E.H., Oxnard, C.E., 1963. The musculature of the primate shoulder. Trans. Zool. Soc. Lond. 29, 553-650.

Ashton, E.H., Oxnard, C.E., 1964. Locomotor patterns in primates. Proc. Zool. Soc. Lond. 142, 1-28.

Boule, M., 1912. L'Homme fossile de la Chapelle-aux-Saints. Ann. Paleontol. 7, 21-192. 
Carretero, J.M., Lorenzo, C., Arsuaga, J.L., 1999. Axial and appendicular skeleton of Homo antecessor. J. Hum. Evol. 37, 459-499.

Cave, A.J.E., 1961. Nature and morphology of the costoclavicular ligament. J. Anat. 95 (2), 170-179.

Fleagle, J.G., 1974. Dynamics of brachiating siamang Hylobates (Symphalangus) syndactylus. Nature 248, 259-260.

Fleagle, J.G., 1978. Mechanical function of primate clavicles (abstract). Am. J. Phys. Anthrop. 48, 394.

Gagey, O., 1985. Etude de l'élévation du membre supérieur. Rôle des ligaments articulaires et des muscles fléchisseurs de l'articulation scapulo-humérale. Mémoires du Laboratoire d'Anatomie de la Faculté de Médecine de Paris 76, 115 p.

Groves, C.P., 1993a. Primates. In: Wilson, D.E., Reeder, D.A. (Eds.), Mammal species of the World, a taxonomic and geographic reference, second edition. Smithsonian Institution Press, Washington and London, $1206 \mathrm{p}$.

Groves, C.P., 1993b. Speciation in living hominoid primates. In: Kimbel, W.H., Martin, L.B. (Eds.), Species, Species Concepts and Primate Evolution. Plenum Press, New York and London, $560 \mathrm{p}$.

Harrington, M.A., Keller, T.S., Seiler, J.G., Weikert, D.R., Moeljanto, E., Schwartz, H.S., 1993. Geometric properties and the predicted mechanical behavior of adult human clavicles. J. Biomechanics 26, 417-426.

Heim, J.L., 1974. Les Hommes fossiles de la Ferrassie (Dordogne) et le problème de la définition des Néandertaliens classiques, L'Anthropologie 78 (1), 81-112.

Heim, J.L., 1976. Les hommes fossiles de La Ferrassie I. Arch. Inst. Paléont. Hum. 35, 1331.

Heim, J.L., 1982a. Les hommes fossiles de la Ferrassie II. Arch. Inst. Paléont. hum. 38, 1272.

Heim, J.L., 1982b. Les enfants néandertaliens de la Ferrassie. Etude anthropologique et analyse ontogénique des hommes de néandertal. Masson, Paris, 169 p.

Jenkins, F.A. Jr., 1974. The movement of the shoulder in claviculate and aclaviculate Mammals. J. Morph. 144, 71-84.
Jenkins, F.A., Dumbrowski, P.J., Gordon, E.P., 1978. Analysis of the shoulder in brachiating spider monkeys (Ateles geoffroyi). Am. J. Phys. Anthrop. 48, 65-75.

Jit, I., Kaur, H., 1986. Rhomboid fossa in the clavicles of North Indians. Am. J. Phys. Anthrop. 70, 97-103.

Jouffroy, F.K., 1962. La musculature des membres chez les Lémuriens de Madagascar. Etude descriptive et comparative. Mammalia 26, suppl. 2, 322 p.

Kapandji, I.A., 1994. Physiologie articulaire. $\mathrm{tl}$ Membre supérieur. 5ème édition, Maloine, Paris, 296 p.

Kleiweg de Zwaan, J.P., 1931. La clavicule des javanais de l'est de Java. L'Anthropologie 41, 273-287.

Konstant, W., Stern, J.T. Jr, Fleagle, J.G., Jungers, W.L., 1982. Function of the subclavius muscle in a nonhuman primate, the spider monkey (Ateles). Folia Primatol. (Basel) 38 (3-4), 170-182.

Larson, S.G., Stern, J.T. Jr., Jungers, W.L., 1991. EMG of serratus anterior and trapezius in the chimpanzee: scapular rotator revisited. Am. J. Phys. Anthrop. 85, 71-84.

Leakey, R.E.F., Walker, A.C., 1985. Further Hominids from the Plio-Pleistocene of Koobi Fora, Kenya. Am. J. Phys. Anthrop. 67, 135163.

Ljunggren, A.E., 1979. Clavicular function, Acta. Orthop. Scand. 50, 261-268.

Marchal, F., 2000a. A new morphometric analysis of the hominid pelvic bone. J. Hum. Evol. 38, 347-365.

Marchal, F., 2000b. L'ischion et le pubis des néandertaliens : Morphologie particulière ou héritage ancestral ? Biom. Hum. et Anthropol. 18, 77-85.

Matiegka, J., 1938. La déflexion de la clavicule. L'Anthropologie 48, 596-597.

Martin, C.P., O'Brien, H.D., 1939. The coracoid process in the primate. J. Anat. and Physiol. $73,630-642$.

Mays, S., Steele, J., Ford, M., 1999. Directional asymmetry in the human clavicle. Int. J. Osteoarchaeol. 9, 18-28.

Napier, J.R., 1965. Réponse à Tobias, New discoveries in Tanganika, their bearing on hominid evolution. Curr. Anthrop. 6, 402-403. 
Nara, T., 1994. Etude de la variabilité de certains caractères métriques et morphologiques des néandertaliens. Thèse de doctorat, Université de Bordeaux I, $212 \mathrm{p}$.

Olivier, G., 1951a. Technique de mesure des courbures de la clavicule. C.R. Ass. Anat., XXXIXème Réunion (Nancy) 69, 753-764.

Olivier, G., 1951b. Anthropologie de la clavicule. Bull. et Mém. de la Soc. Anthrop. Paris 2 (10), 67-99, 121-157.

Olivier, G., 1953. La clavicule du Semnopithèque. Mammalia XVII (3), 173-186.

Olivier, G., 1954. Anthropologie de la clavicule. Bull. et Mém. de la Soc. Anthrop. Paris V (10), 144-153.

Olivier, G., 1955. Anthropologie de la clavicule. Bull. et Mém. de la Soc. Anthrop. Paris VI (10), 282-302.

Olivier, G., 1965. Anatomie anthropologique. Vigot édition, Paris, $162 \mathrm{p}$.

Olivier, G., Capliez, S., 1957. Anthropologie de la clavicule. Bull. et Mém. de la Soc. Anthrop. Paris VIII (10), 225-261.

Olivier, G., Chabeuf, M., Laluque, P., 1954. Anthropologie de la clavicule. Bull. et Mém. de la Soc. Anthrop. Paris V (10), 35-46.

Parson, F.G., 1917. On the modern english clavicle. J. Anat. and Physiol. 51, 71-93.

Patte, E., 1955. Les Néandertaliens. Masson, Paris, $559 \mathrm{p}$.

Ray, L.J., 1959. Metrical and non-metrical features of the calvicle of the Australian Aboriginal. Am. J. Phys. Anthrop. 17, 217-226.

Sakka, M., 1985. Morphologie évolutive de la tête et du cou chez l'Homme et les Grands Singes. Application aux Hominidés fossiles, t.I ensembles anatomiques et cervicaux. Cahiers de Paléoanthropologie, CNRS, Paris, $168 \mathrm{p}$.

Sankhyan, A.R., 1997. Fossil clavicle of a Middle Pleistocene hominid from the central Narmada Valley, India. J. Hum. Evol. 32, 3-16.
Schultz, A.H., 1930. The skeleton of the trunk and limbs of higer primates. Hum. Biol. II (3), 303-438.

Schultz, A.H., 1950. The physical distinction of Man. Proc. Am. Phil. Soc. 94, 428-449.

Spoor, F., Wood, W., 1999. Neck proportion in modern humans and Neanderthals. Am. J. Phys. Anthrop., (abstract), suppl. 28, 256.

Sullivan, W.E., Osgood, C.W., 1927. The musculature of the superior extremity of the Orang-utan. Anat. Rec. 35, 193-239.

Terry, R.J., 1932. The clavicle of the American Negro. Am. J. Phys. Anthrop. 16, 351-379.

Vandermeersch, B., 1981. Les Hommes fossiles de Qafzeh (Israël). Cahiers de Paléoanthropologie, C.N.R.S, Paris, 308 p.

Vandermeersch, B., Trinkaus, E., 1995. The postcranial remains of the Regourdou 1 Neandertal: the shoulder and arm remains. J. Hum. Evol. 28, 439-476.

Vallois, H.V., 1928. L'omoplate humaine. Etude anatomique et anthropologique. Bull. et Mém. de la Soc. Anthrop. Paris, 7 (IX), 129168.

Voisin, J.L., 2000a. L'épaule des hominidés. Aspects architecturaux et fonctionnels, références particulières à la clavicule. Thèse $\mathrm{du} \mathrm{Mu-}$ séum National d'Histoire Naturelle, 442 p. 2 vols.

Voisin, J.L., 2000b. La clavicule humaine : adaptation à la station érigée ?, Biom. Hum. et Anthropol. 18, 15-22.

Voisin, J.L., 2000c. Les clavicules néandertaliennes : reflet d'un complexe scapulaire particulier ?; Acte du colloque : L'identité humaine en question, mai 1999 édition ARTCOM, Paris, 80-91.

Walker, A., Leakey, R., 1993. Clavicles In: Walker, A., Leakey, R. (Eds.), The Narikotome Homo enectus skeleton. Springer-Verlag (Berlin), 457 p. 